\title{
Bericht aus den EAP-Meetings und vom 23. Kongress der EAP in Belgrad
}

Peter Schulthess

Die Herbst-EAP-Meetings fanden vom 18.20. Oktober 2018 in Belgrad statt. Zeitlich überschneidend fand vom 18.-21. Oktober der 23. EAP Fach-Kongress statt, der zugleich der 8. Kongress der Serbischen Vereinigung für Psychotherapie war.

\section{Die EAP hat einen neuen Рräsidenten}

Charles Cassar aus Malta hat als Vizepräsident statutengemäss bereits etwas vorzeitig das Amt des Präsidenten übernommen, nachdem der aktuelle Präsident, Philippe Vranken aus Belgien wegen eines Konfliktes mit dem Vorstand sein Amt Anfang Mai abgab. Seine Amtsdauer hätte noch bis Februar 2019 gedauert. In einem Brief an die Mitglieder des erweiterten Vorstandes teilte er dies mit, ohne dass ersichtlich wurde, worum es im Konflikt wirklich ging. Befürchtungen, dass ein Teil des exekutiven Vorstandes ihm politisch nicht folgen wollte, die Beschlüsse des erweiterten Vorstandes umzusetzen, auch bezüglich Lobbying, erwiesen sich als unbegründet. Trotz Aussprache am Anfang der Belgrader Meetings blieben die Gründe für den Rücktritt im Konflikt im Unklaren.

Philippe Vranken war ein sehr initiativer Präsident, der viele Änderungen in der EAP anstieß und deshalb für viele als Hoffnungsträger galt Repräsentanten der althergebrachten Machtstrukturen sahen das nicht gerne und versuchten den Präsidenten zurückzubinden.

Auch wenn er nun vorzeitig zurücktrat, so hat er grosse Verdienste an einer Vitalisierung der EAP. Charles Cassar beabsichtigt, die von Philippe Vranken eingeleiteten Reformen und Aktivitäten weiter voranzutreiben und insbesondere auf der EU-Bühne politisch weiterhin aktiv zu sein für unseren Berufsstand. Er hat grosse Verdienste daran, dass in Malta ein Psychotherapiegesetz verabschiedet wurde, welches den PsychotherapeutInnenberuf nicht als psychologischen oder medizinischen Beruf versteht, sondern als eigenständigen wissenschaftlichen Beruf in eigenen Rechten.

\section{Neue Psychotherapiegesetze}

in Malta und Kroatien

In beiden Ländern sind im Verlaufe des Jahres Gesetze in Kraft getreten, welche Psychothera- pie als eigenständigen Beruf regeln. Das ist ein wichtiger Fortschritt und Erfolg auch für die EAP. Verbunden damit ist eine Reform im Bildungswesen der höheren Schulen. Es ist nun nicht nur möglich, einen universitären $\mathrm{Ab}$ schluss als Master oder PhD in Psychotherapie zu machen, sondern Weiterbildungsinstitute haben die Möglichkeit, sich als praxisbezogene Hochschulen anerkennen zu lassen (das entspricht bei uns den Fachhochschulen) und Master-, wie auch Doktoratsprogramme anzubieten. So wird es in Malta künftig möglich sein, einen Master oder einen PhD in Gestalttherapie zu erwerben, da das dortige Gestalttherapie Institut die Anerkennung als Fachhochschule geschafft hat. Solche Programme (unterschiedlicher Therapierichtungen) existieren schon länger im angelsächsischen Raum als Kooperationsprojekte privater Weiterbildungsinstitute mit Universitäten.

\section{Tagungsberichte}

Der Vorsitzende des Science and Research Committee (SARC), Peter Schulthess, berichtete über die drei seitens des SARC in diesem Jahr besuchten wissenschaftlichen Kongresse. Am Kongress der Europäischen Vereinigung für Psychiatrie (EPA) zeigte sich, dass Psychotherapie und Psychotherapieforschung kaum ein Thema waren. Die Präsentationen betrafen psychiatrische, neurowissenschaftliche und pharmakologische Themen. Der Vorstand der EPA ist allerdings daran interessiert, seine Mitglieder mehr über Psychotherapie wissen zu lassen und sucht die Kooperation und Mitwirkung der EAP an den Kongressen.

Über die Kongresse der International Federation for Psychotherapy (IFP) und Society for Psychotherapy Reserach (SPR) ist in der Zeitschrift Psychotherapie-Wissenschaft, Heft 2-2018, ausführlich berichtet worden. Dem Board wurden ebenfalls ausgewählte Erkenntnisse zum Stand der Diskussion in der Forschung weitergegeben. Die Mitglieder des erweiterten EAP Vorstandes zeigten sich dankbar für diese Berichte. Es wurde gewünscht, dass die EAP Kongressdaten auf der Website aufschaltet (nicht nur die eigenen), wie auch Links zu führenden Journals.

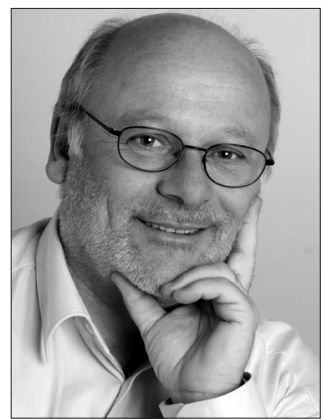


Neues Sekretariat

und Neues Finanzkomitee

Die Geschäftsstelle der EAP ist aus Kostengründen umgezogen. Neu befindet sie sich an der Mariahilfer Straße 1d/3. Stock/Tür 13, 1060 Wien.

Die Finanzen sind ein aktuelles Thema in der EAP: Die Einnahmen gehen zurück, die Ausgaben steigen. Das muss besser in den Griff bekommen werden, durch Schaffung von Möglichkeiten zu Mehreinnahmen und durch Senkung der Kosten. Neu wurde eine Arbeitsgruppe eingesetzt, welche als Finanzkomitee wirken soll und entsprechende Ideen vertieft bespricht als konsultatives Organ für den Kassier und das Executive Committee.

\section{Kammer für Weiterbildungsinstitute}

$\mathrm{Zu}$ zweiten Mal fand auch eine Sitzung der VertreterInnen der EAP-akkreditierten Weiterbildungsinstitute statt. Das gab dem SARC die Gelegenheit, die Studie SPRISTAD der SPR nochmals zu präsentieren und die Weiterbildungsinstitute zur Mitwirkung zu motivieren. SPRISTAD untersucht die berufliche und persönliche Entwicklung von WeiterbildungsteilnehmerInnen im Verlaufe ihres Trainings. Ausserdem gab dies die Gelegenheit, sich mit der Weiterentwicklung der Programme zu befassen, sodass sie den Bildungslevel 7 erreichen (entspricht einem Master). Dazu muss auch ein Forschungsmodul Teil der Weiterbildungsprogramme werden. Dieses Thema soll an den nächsten Meetings mit einer ganztätigen Veranstaltung weiter vertieft werden. In der laufenden Statutenrevision sollen die Weiterbildungsinstitute eine eigene Kammer erhalten.
Dokument zum Brexit aus Sicht der PsychotherapeutInnen

Auf Wunsch der Präsidentin der Britischen Vereinigung für Psychotherapie (UKCP) trafen sich Interessierte zu einer separaten Sitzung, um sich auszutauschen über den bevorstehenden Brexit und was das für Auswirkungen auf die PsychotherapeutInnen, die Ausbildung und PatientInnen hat. Es wurden Aspekte gesammelt, die im Nachgang zu den Meetings zu einem Dokument ausgearbeitet werden sollen, welches auch politisch auf der Ebene der EU und britischen ParlamentarierInnen zu Verfügung gestellt werden soll.

\section{Erfolgreiche Tagung}

Gegen 500 Teilnehmende trafen zum eingangs erwähnten parallel laufenden Kongress ein. Das Tagungsthema lautete: «Sense and Sensitivity in Psychotherapy». Viele Fachreferate und Workshops auf Serbisch wie auch auf Englisch wurden angeboten. Die EAP-Kongresse geben dem veranstaltenden Landesverband immer auch die Möglichkeit, durch Einladung von ReferentInnen der EAP-Gremien, die europäische Einbindung des Landesverbandes für seine Mitglieder sichtbar zu machen. Umgekehrt kann sich die EAP den Mitgliedern eines Landesverbandes darstellen mit ihren Leistungen für den Beruf der Psychotherapie. So nutzte auch das SARC die Gelegenheit, über «Sense and Sensitivity in Psychotherapy Research» zu berichten, um PraktikerInnen zur Partizipation an Forschung zu motivieren.

Peter Schulthess ist Vorstandmitglied der ASP und vertritt diese gemeinsam mit Gabriela Rüttimann in der EAP. 